\title{
METAIS PESADOS NOS SEDIMENTOS DE CORRENTE, COMO INDICADORES DE RISCOAMBIENTAL - VALE DO RIO DOS SINOS, RS
}

Luis Eduardo ROBAINA

Milton Luis L. FORMOSO

Carlos Alberto da F. PIRES

\section{RESUMO}

A área de pesquisa representa uma das regiões do Rio Grande do Sul, com intensas atividades industriais e ocupação urbana. Os metais analisados foram $\mathrm{Cu}, \mathrm{Cr}, \mathrm{Cd}$, $\mathrm{Pb}, \mathrm{Zn}$ e Ni. A análise da poluição foi realizada através de quatro relações: concentração total de metais traços nos sedimentos e o nível de base estabelecido nesse estudo; concentração de metais traços na fração disponível e o nível de base; concentração total de metais traços nos sedimentos e os teores médios, conforme dados apresentados na literatura; determinação do Índice de Geoacumulação. Este estudo revela que os processos de urbanização e industrialização estão associados com alta concentração de metais pesados. Os metais $\mathrm{Cr}$, Cu e Zn apresentam risco de muito alto a extremo em algumas drenagens da área. Para a despoluição do rio dos Sinos e seus tributários no futuro é necessário tratar os efluentes antes de chegarem às drenagens.

Palavras-chave: poluição, metais pesados, geoquímica.

\section{ABSTRACT}

The studied area is one of the most intensively populated ones of Rio Grande do Sul with intensive industrial activities. The heavy metals analysed were $\mathrm{Cr}, \mathrm{Pb}, \mathrm{Cu}, \mathrm{Zn}$, $\mathrm{Ni}$ and $\mathrm{Cd}$. Pollution analysis was performed by comparing: metal total concentrations in sediments and local background; metal concentration in mobile phases and local background; total metal concentrations and metal levels reported in the literature and in the Geoaccumulation Index. The study reveals that the urbanization and industrialization processes are associated with higher concentrations of heavy metals. $\mathrm{Cr}, \mathrm{Cu}$ and $\mathrm{Zn}$ have higher concentrations in the sediments of the region. To keep the Sinos River and tributaries clean for the future, it is strongly recommended that urban and industrial effluents should be treated before discharge into the river.

Keywords: pollution, heavy metals, geochemistry

\section{INTRODUÇÃO}

No ambiente aquático, o compartimento considerado mais significativo na concentração de metais é representado pelos sedimentos (AXTMANN \& LUOMA 1991) e, por esse motivo, são muito utilizados como material de amostragem, tanto em pesquisa de prospecção mineral como em estudos ambientais. Ao refletirem a qualidade do sistema e o desenvolvimento histórico de parâmetros hidrológicos e químicos (SALOMONS \& FÖRSTNER 1984) são particularmente úteis na identificação, no monitoramento e no controle de fontes de poluição.
Com relação às fontes antrópicas, vários estudos têm registrado elevada concentração de metais nos sedimentos dos rios, causada por fontes industriais (RAMAMOORTHY \& RUST 1978, RULE 1986, SINGH et al. 1997, entre outros). Além dessas, os esgotos domésticos são importantes fontes de metais (RUBIN 1976, NTEKIM et al. 1993).

No Brasil, mais especificamente no Rio Grande do Sul, merecem destaque estudos do Departamento Municipal de Águas e Esgotos de Porto Alegre (DMAE 1975) com estudos nos rios Gravataí, Sinos e Guaíba, da rede de monitoramento da Fundação Estadual de Proteção Ambiental (FEPAM 1995), de BAISCH na Laguna dos Patos, com tese de doutora- 
do em 1994 e por HATJE et al. (1998), que estimou a concentração de metais pesados nas águas.

A área de pesquisa representa uma das regiões do Estado do Rio Grande do Sul com maior número de atividades industriais e com elevada ocupação urbana. Localizada no Vale do Rio do Sinos, está limitada geograficamente pelas coordenadas: $29^{\circ} 50^{\prime}$ e 29³6' de latitude sul e 51²12' e 5057’ de longitude oeste, abrangendo principalmente os municípios de Campo Bom, Novo Hamburgo e São Leopoldo (Figura 1).

Este estudo objetiva apresentar uma análise de poluição por metais pesados nos sedimentos de corrente no Vale dos Sinos.

\section{METODOLOGIA}

Os metais escolhidos para análise foram $\mathrm{Cu}$, $\mathrm{Cr}, \mathrm{Cd}, \mathrm{Pb}, \mathrm{Zn}$ e Ni, baseado nos seguintes parâmetros: toxicidade potencial para o homem e para a biota aquática, porque são os mais freqüentemente lançados pelas empresas situadas na região, e são os metais analisados em outros estudos realizados na região, especialmente pela FEPAM.

Os pontos de amostragem foram registrados de acordo com seus posicionamentos (latitudes e longitudes), verificados em trabalhos de campo através do instrumento de localização GPS (Global Position System). A malha de coleta foi adensada

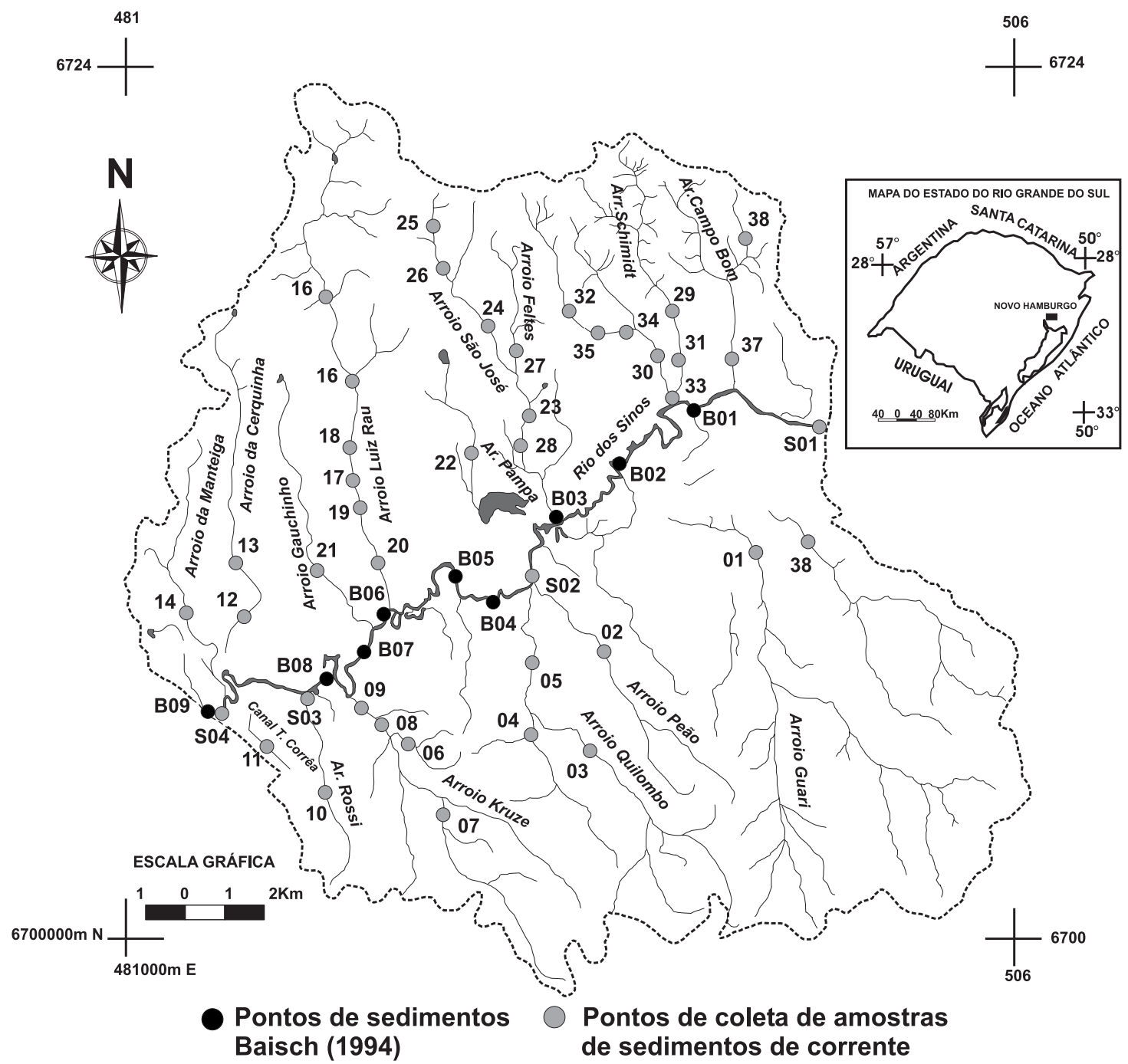

FIGURA 1 - Mapa de localização dos pontos de coleta de sedimentos de corrente. 
nas áreas urbanas, prevendo avaliar a localização de fontes de poluição. Para que a amostra fosse o mais representativa possível da área de captação a montante, a coleta foi feita na calha da drenagem em três posições, abaixo do nível d’água, nas áreas de deposição (ex. porção côncava da curva, encontro de duas correntes d’água, etc).

A coleta de sedimento de fundo foi feita manualmente, por coletor construído com canos de PVC, $100 \mathrm{ml}$, na forma de " $\mathrm{L}$ ", diretamente no fundo da drenagem. Coletou-se aproximadamente $1 \mathrm{~kg}$ de sedimento por estação, separando-se a fração < 0,062mm por peneiração a úmido. A fração $<0,062 \mathrm{~mm}$ foi escolhida por 4 motivos: metais pesados têm sido encontrados principalmente na fração silto-argilosa; essa fração é mais próxima da granulometria carregada em suspensão (que é o mais importante transporte do sedimento); a peneira não altera as concentrações metálicas por remobilização, principalmente quando se utiliza a água do mesmo sistema; numerosos estudos sobre metais já têm sido feitos utilizando a referida fração.

Para comparar a variação da concentração provocada pelas condições hidrodinâmicas nas drenagens afluentes do Rio dos Sinos realizou-se amostragem, em alguns pontos, em dois períodos, período seco (condição de nível baixo) e período chuvoso (nível alto), procurando manter a mesma região de amostragem. Períodos secos e chuvosos foram estabelecidos com base nas descargas do Rio dos Sinos e cotas estabelecidas na estação de coleta no município de São Leopoldo, conforme dados do Departamento Nacional de Água, Esgoto e Energia. As coletas do mês de janeiro, período seco, apresentam uma média para a vazão de $34 \mathrm{~m}^{3} / \mathrm{s}$ e nível médio ao redor de $170 \mathrm{~cm}$; no mês de outubro foram feitas as coletas que representam o período chuvoso. Neste período a vazão ocorre em média superior a $60 \mathrm{~m}^{3} / \mathrm{s}$ e com cotas medidas na régua na estação de coleta de São Leopoldo ao redor de $400 \mathrm{~cm}$, ou seja, duas vezes superior às anteriores.

No Rio dos Sinos a coleta foi feita por arrasto de um recipiente de alumínio com peso de $10 \mathrm{~kg}$, colocado na parte lateral de uma embarcação.

Como apoio para as análises e interpretações, identificou-se o conteúdo de carbono orgânico (CO) e o $\mathrm{pH}$.

Trabalhou-se com uma fração que denominamos de disponível e outra, fração total. Para extração da fração disponível utilizou-se o meio extrator recomendado por MORSCH (1991), que testou vários extratores e optou pela utilização de $\mathrm{HNO}_{3} 0,5 \mathrm{~N}+$ $\mathrm{H}_{2} \mathrm{O}_{2}$ a $30 \%$, segundo a autora, pela melhor adequa- ção a análise por EAA, com menor efeito matriz. Além disso, a condição urbana, da região pesquisada, gera uma forte presença orgânica nos sedimentos e, ainda, conforme BAISCH (1994), a fase oxidável é bastante representativa no Rio dos Sinos. A digestão ácida foi realizada com proporção de $2,5 \mathrm{~g}$ de amostra para $40 \mathrm{ml}$ de ácido e $1 \mathrm{ml}$ de $\mathrm{H}_{2} \mathrm{O}_{2}$ a $30 \%$, deixando extrair sob periódica agitação durante $3 \mathrm{~h}$, com temperatura ao redor de $60^{\circ} \mathrm{C}$. Após, as amostras foram retirados em papel filtro, aferidas ao volume de $50 \mathrm{ml}$ e armazenadas em frasco de polietileno para posterior análise.

Na abertura total, além da análise de solos e rochas, utilizou-se a técnica empregada no laboratório de análise do Instituto de Geociências da Universidade Federal do Rio Grande do Sul (UFRGS). Uma primeira fase emprega $\mathrm{HF}: \mathrm{HNO}_{3}$, na relação 2:1, depois adiciona-se a mistura de ácidos $\mathrm{HClO}_{4}: \mathrm{HNO}_{3}: \mathrm{HF}$ na proporção 1:1:1 e, por fim, $\mathrm{HCl}(6 \mathrm{M})$.

\section{CARACTERÍSTICAS DOS SEDIMENTOS DAS DRENAGENS DA REGIÃO}

O mapa da figura 2 mostra os pontos de coleta nas drenagens da área de estudo.

As drenagens da área, afluentes do Rio dos Sinos, caracterizam-se por apresentarem calhas que variam de $2 \mathrm{~m}$ a $5 \mathrm{~m}$ de diâmetro e profundidade em geral inferior a $1 \mathrm{~m}$. Os sedimentos predominantes são tamanho areia.

$\mathrm{O}$ pH dos sedimentos da região varia entre 5 e 6,5, valores comuns em áreas continentais.

O carbono orgânico apresenta valores entre 3 e $4 \%$ nas drenagens de áreas urbanas. Nas drenagens cujas margens mantém alguma vegetação, os valores de carbono orgânico são relativamente maiores, como é o caso dos arroios Peão, Guari e Campo Bom. O arroio Schmidt, que cruza uma área de parque no município de Campo Bom, também apresenta valores relativamente elevados.

Mineralogicamente, o quartzo é o constituinte mais importante, ocorrendo secundariamente óxidos de ferro e feldspato. Na fração fina o argilomineral do grupo da caolinita é o constituinte essencial, ocorrendo em pequena proporção argilomineral 2:1, classificado como camadas mistas de ilita-esmectita.

No Rio dos Sinos as frações granulométricas são variáveis. Os sedimentos apresentam valores de Eh negativo, caracterizando condições reduzidas do fundo (BAISCH 1994). Os valores de $\mathrm{pH}$ variam de 6 a 7,5, com predominância de condições levemente ácidas. A concentração de matéria orgânica é elevada, estando fortemente associada, conforme 
BAISCH (1994), à contribuição antrópica. Os minerais da fração fina são a caolinita e, em proporção elevada, argilomineral do tipo 2:1, classificado como do grupo das esmectitas.

As concentrações dos seis metais pesados, estudadas nos sedimentos de corrente das drenagens da área, estão apresentadas nas figuras 3 e 4. As análises estabeleceram períodos de baixas cotas fluviais dos arroios, onde os valores são concentrados, estando representados pela letra (s), e períodos de cotas fluviométricas elevadas, indicados pela simbologia (u). Além disso, como explicitado na metodologia, foram medidas concentrações totais, que estão indicadas por (T), e concentrações obtidas na fração disponível, por (D). A concentração de Cd refere-se somente ao total em condições úmidas.

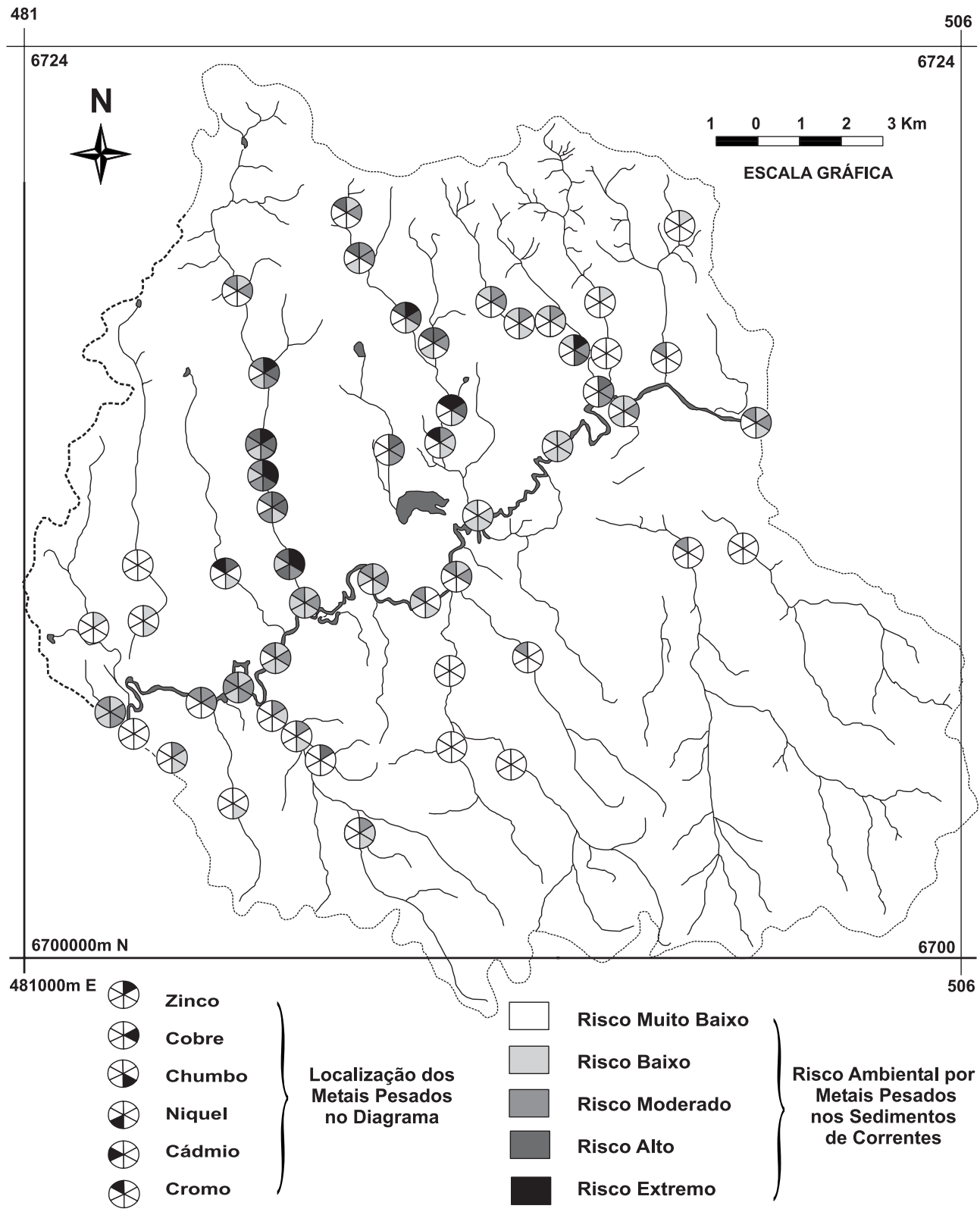

FIGURA 2 - Risco ambiental por metais pesados nos sedimentos de corrente. 


\section{DETERMINAÇÃO DO NÍVEL DE BASE}

Os cursos d'água ao cruzarem as rochas e solos carregam elementos químicos e partículas destes, que irão depositar-se junto aos sedimentos do rio. Dessa forma, os sedimentos dos rios representam uma amostragem química das áreas drenadas por seu curso.

O nível de base foi estabelecido baseado nos teores médios definidos para as rochas e para os solos. Os limites das rochas e solos são determinados pela soma da média mais duas vezes o desvio padrão. A média entre os valores obtidos dará o nível de base para os sedimentos dos rios (Quadro 1).

A equação que define os teores da margem esquerda é representada pela média entre os teores limites medidos nas rochas sedimentares e nos argissolos:

$$
-[\operatorname{trs}+\mathrm{tsp}] / 2
$$
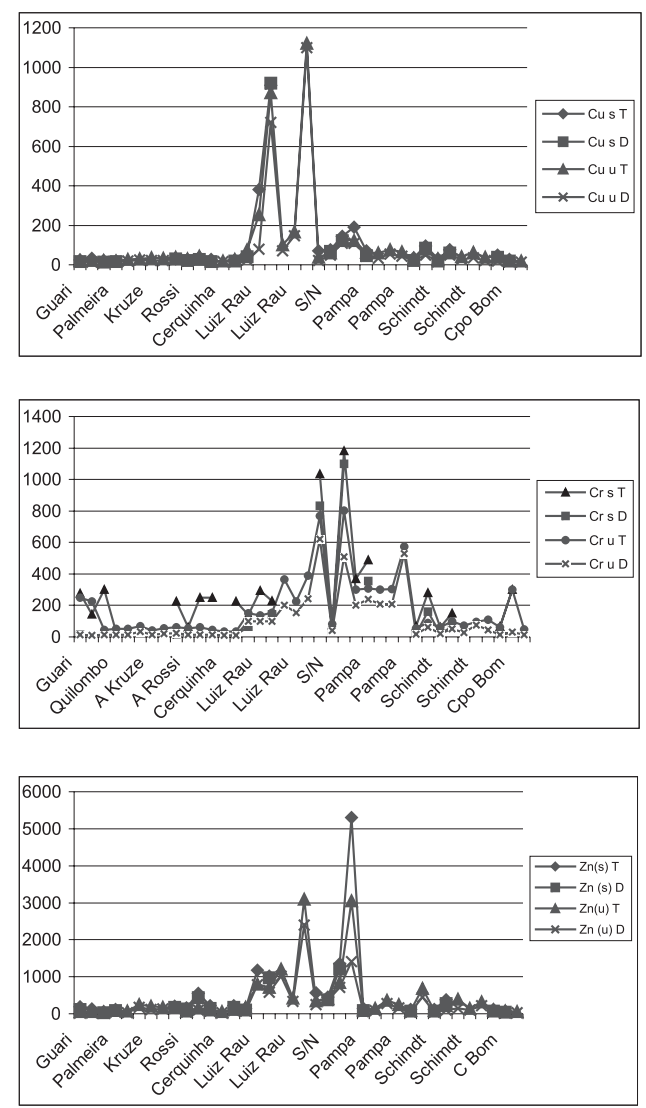

Na margem direita do Rio dos Sinos, além das rochas sedimentares, seqüências vulcânicas interferem nos teores. A participação de cada uma depende da área ocupada na bacia. Estabelecemos de forma geral para as drenagens da margem direita uma relação de 1:5 entre os terrenos vulcânicos e sedimentares.

Dessa forma, a equação ficou definida por:

$\{\{[\operatorname{trs}+\mathrm{tsp}] / 2\} .5+\{[\operatorname{trv}+\operatorname{tav}] / 2\} .1\} / 6$,

onde trs representa o teor limite da rocha sedimentar, tsp o teor limite dos solos, trv o teor limite das rochas vulcânicas e tav, o teor limite da rocha vulcânica alterada.

Utilizando as equações temos os teores de nível de base para cada elemento, em ambas as margens (Quadro 2).

O teor limite de $\mathrm{Cd}$ foi estabelecido com base nos teores obtidos nas rochas sedimentares da área, de 0,54ppm, para ambas as margens.
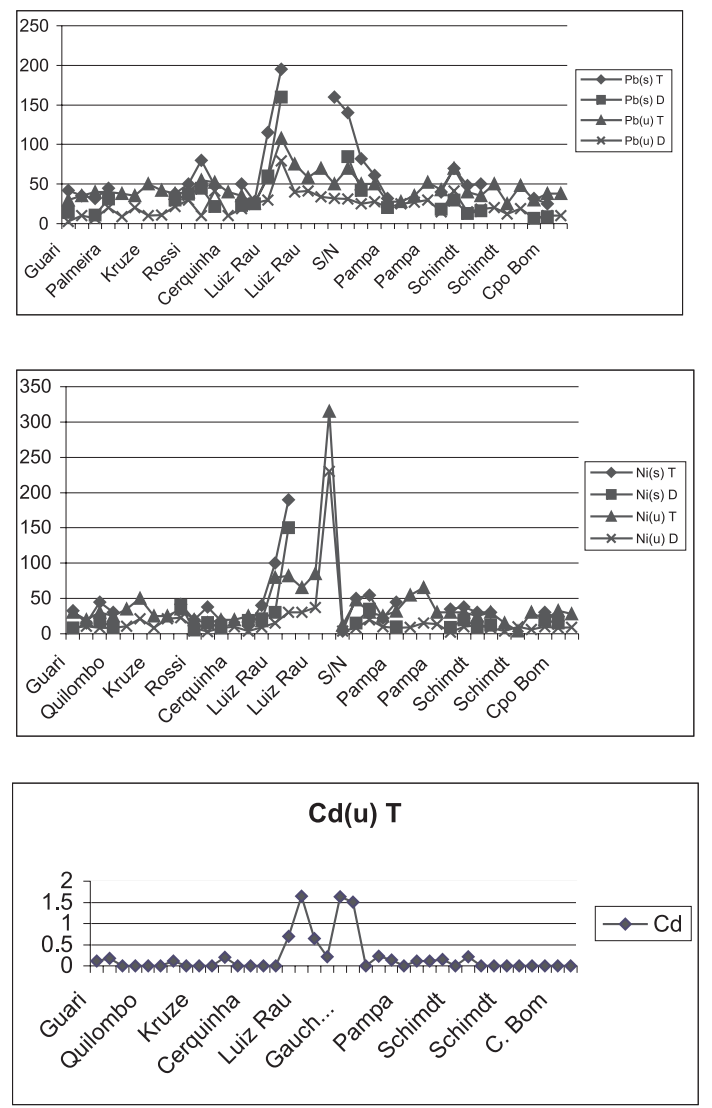

FIGURA 3 - Concentração de metais pesados (Cu, Cr, Zn, Pb, Ni, Cd) em ppm nos sedimentos de corrente nos afluentes da área de estudo ( - coletas em períodos secos; u - coleta em período úmido; T - concentração total; D concentração disponível). 

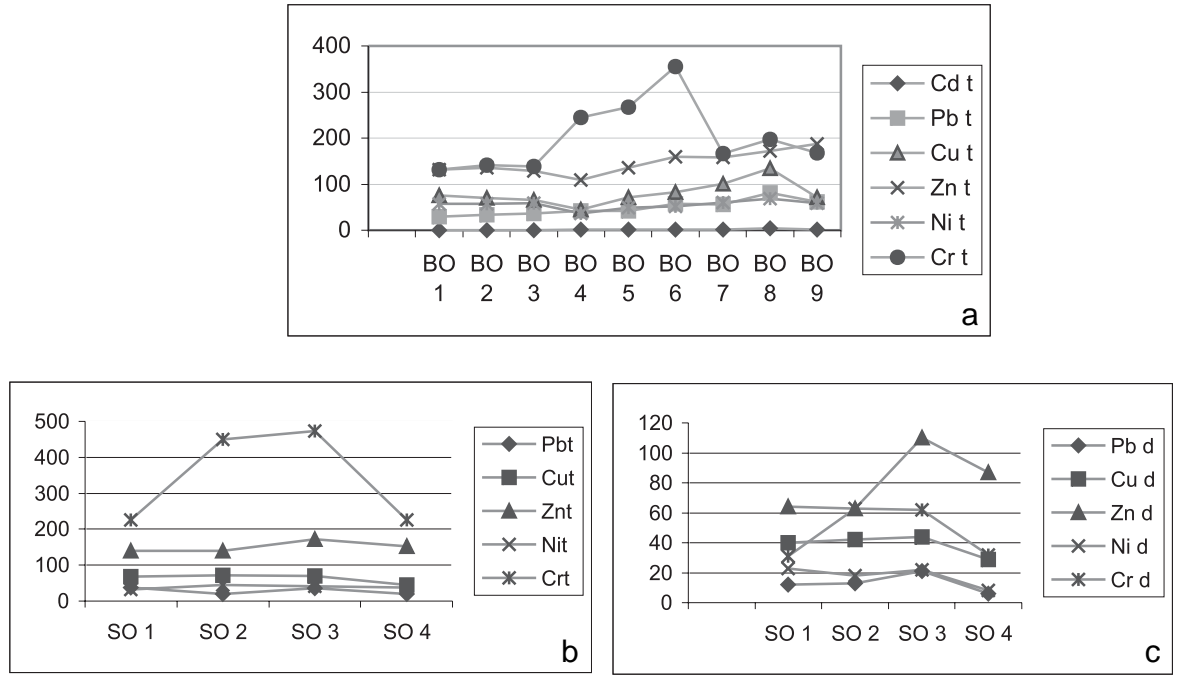

FIGURA 4 - Concentração de metais (ppm) nos sedimentos do rio dos Sinos. a) concentração total nas estações de amostragem BO1 a BO9; b) concentração total nas estações de amostragem SO1 a SO4; c) concentração disponível nas estações de amostragem SO1 a SO4.

QUADRO 1 - Teores limites (ppm) definidos pelas análises de rochas e produtos de alteração medidas neste estudo.

\begin{tabular}{|ccccc|}
\hline $\begin{array}{c}\text { Elementos/ } \\
\text { Teores }\end{array}$ & $\begin{array}{c}\text { Teor limite } \\
\text { rochas sedimentares }\end{array}$ & $\begin{array}{c}\text { Teor limite } \\
\text { argissolos }\end{array}$ & $\begin{array}{c}\text { Teor limite } \\
\text { rochas vulcânicas }\end{array}$ & $\begin{array}{c}\text { Teor limite } \\
\text { alteração de } \\
\text { rochas vulcânicas }\end{array}$ \\
\hline $\mathrm{Pb}$ & 36,08 & 51,81 & 10 & 30,74 \\
$\mathrm{Cu}$ & 31,92 & 33,5 & 107,36 & 120,22 \\
$\mathrm{Zn}$ & 77,65 & 81,32 & 116,52 & 11,9 \\
$\mathrm{Ni}$ & 45,79 & 49,29 & 128 & 38,15 \\
$\mathrm{Gr}$ & 74,17 & 81,2 & 154,04 & 63,41 \\
$\mathrm{Cd}$ & 0,54 & 0,54 & & \\
\hline
\end{tabular}

\section{ANÁLISE DO GRAU DE POLUIÇÃO}

Na análise da poluição utilizaram-se comparações entre os teores medidos nos sedimentos e valores estabelecidos como naturais, ou não poluídos. Dessa forma, obtivemos quatro relações assim definidas:

A - Relação entre a concentração total de metais traços nos sedimentos e o nível de base estabelecido nesse estudo, conforme a fórmula:

$$
\begin{aligned}
\mathrm{I}= & \mathrm{Ct} /(\mathrm{X}+2 \&) \\
& \mathrm{Ct}-\text { Concentração total } \\
& \mathrm{X} \text { - Média } \\
& \& \text { - Desvio padrão } \\
& \mathrm{X}+2 \& \text { - Nível de base }
\end{aligned}
$$

$B$ - Relação entre a concentração de metais traços na fração disponível, dos sedimentos e o nível de base estabelecido nesse estudo. A fração disponível representa o principal risco ambiental, devido à mobilidade potencial. Diversos estudos têm demonstrado que, em sedimentos não poluídos ou com baixa contribuição antrópica, a concentração de metais na fração disponível é inferior a 50\% da concentração total (PESTANA 1989, FÖRSTNER \& WITTMAN 1983). Dessa forma, estabelecemos esse valor como índice para estabelecer as análises comparativas.

Nessa relação, para estabelecermos os graus de poluição, comparamos o teor total com o nível de base, a participação da fração disponível na concentração total, se $>$ ou $<50 \%$, e a relação entre a fração disponível e o nível de base. 
Revista do Instituto Geológico, São Paulo, 23(2), 35-47, 2002.

QUADRO 2 - Relação dos níveis de base estabelecidos neste estudo para os metais pesados analisados em ppm.

\begin{tabular}{|ccc|}
\hline Metal Pesado & Nível de base margem direita & Nível de base margem esquerda \\
\hline $\mathrm{Pb}$ & $40,0 \mathrm{ppm}$ & $43,9 \mathrm{ppm}$ \\
$\mathrm{Cu}$ & $46,2 \mathrm{ppm}$ & $32,7 \mathrm{ppm}$ \\
$\mathrm{Zn}$ & $85,3 \mathrm{ppm}$ & $79,5 \mathrm{ppm}$ \\
$\mathrm{Ni}$ & $53,5 \mathrm{ppm}$ & $47,5 \mathrm{ppm}$ \\
$\mathrm{Cr}$ & $82,9 \mathrm{ppm}$ & $77,7 \mathrm{ppm}$ \\
$\mathrm{Cd}$ & $0,5 \mathrm{ppm}$ & $0,5 \mathrm{ppm}$ \\
\hline
\end{tabular}

QUADRO 3 - Índices e classes determinados para as relações A e B.

\begin{tabular}{|c|c|c|c|}
\hline$<1$ & $\mathrm{CT}<\mathrm{NB}$ e $\mathrm{FD}<70 \%$ do NB & 0 & Não poluído \\
\hline $1-1,5$ & $\mathrm{CT}>\mathrm{NB} ; \mathrm{CT} / \mathrm{FD}<0,5 ; \mathrm{FD}<70 \%$ do $\mathrm{NB}$ & 1 & moderado/não poluído \\
\hline $1,5-3,0$ & $\mathrm{CT}<$ ou $>\mathrm{NB}$; CT/FD $<0,5 ; \mathrm{FD}>70 \%$ do $\mathrm{NB}$ & 2 & moderadamente poluído \\
\hline $3,0-5,0$ & $\mathrm{CT}>\mathrm{NB} ; \mathrm{CT} / \mathrm{FD}>0,5 ; \mathrm{FD} / \mathrm{NB}$ de $0,5-1$ & 3 & forte/moderadamente poluído \\
\hline $5,0-7,0$ & $\mathrm{CT}>\mathrm{NB} ; \mathrm{CT} / \mathrm{FD}>0,5 ; \mathrm{FD} / \mathrm{NB}$ de $1-1,5$ & 4 & fortemente poluído \\
\hline $7,0-9,0$ & $\mathrm{CT}>\mathrm{NB} ; \mathrm{CT} / \mathrm{FD}>0,5 ; \mathrm{FD} / \mathrm{NB}$ de $1,5-2,0$ & 5 & extremo/fortemente poluído \\
\hline$>9,0$ & $\mathrm{CT}>\mathrm{NB} ; \mathrm{CT} / \mathrm{FD}>0,5 ; \mathrm{FD} / \mathrm{NB}>2,0$ & 6 & extremamente poluído \\
\hline
\end{tabular}

C - Relação entre a concentração total de metais traços nos sedimentos e os teores médios para solos e sedimentos, conforme dados apresentados na literatura.

$D$ - Determinação do Índice de Geoacumulação (MÜLLER 1969 in NTEKIM et al. 1993), que representa o logaritmo na base 2 da concentração total de elemento, dividido pela média do valor dos folhelhos multiplicado pelo fator 1,5.

$\mathrm{IGEO}=\log _{2} \mathrm{CR} / 1,5 \mathrm{xBn}$

onde CR é a concentração do metal no sedimento, Bn é o “Background”, tomado como média folhelhos. $\mathrm{O}$ fator 1,5 é introduzido para incluir qualquer possível variação dos valores de "Background” devido à variação litológica.

Cada relação feita foi expressa em 7 valores numéricos, que indicam classes de poluição. Os quadros 3 e 4 apresentam os índices estabelecidos para as 7 classes definidas nas relações $A, B$, $C$ e $D$.

\section{DETERMINAÇÃO DAS CLASSES}

Os resultados obtidos nas relações determinam as classes, de 0 (não poluído) a 6 (extremamente poluído), para cada elemento nos pontos de coletas de sedimentos. As figuras 5, 6, 7, 8 e 9 apresentam as classes definidas para cada elemento em todos os pontos de análise para as quatro relações determinadas. Para o Rio dos Sinos, os valores obtidos para as classes de níveis de poluição em cada uma das 4 relações estudadas, estão apresentadas na figura 9.

\section{ANÁLISE DE RISCO AMBIENTAL POR POLUIÇÃO DE METAIS PESADOS}

Para análise do risco ambiental utilizou-se o somatório das classes estabelecidas em cada relação estudada, que variam do extremo não poluído, indicado por 0 , ao extremo poluído, indicado por 6 . Dessa forma, o índice máximo é representado por 24 e o mínimo por 0 , sendo dividido em: muito baixo (0 -4), baixo (5 - 9), moderado (10 - 14), alto (15 - 19) e extremo $(20-24)$. No caso das análises utilizarem 3 relações, como acontece com pontos de sedimentos no Rio dos Sinos, e para o elemento Cd, os valores que estabelecem risco ambiental por metal pesado passam a ser representados pelo valor mínimo de 0 e máximo de 18 , sendo dividido em: muito baixo (0 -3), baixo (4 - 7), moderado (8-11), alto $(12-15)$ e extremo $(16-18)$. 
QUADRO 4 - Índices das classes determinadas em ppm para as relações C e D.

\begin{tabular}{|ccccccccl|}
\hline & \multicolumn{3}{c}{ ÍNDICE C } & & & IGEO & CLASSES & Qualidade do sedimento \\
$(\mathrm{Cu})$ & $(\mathrm{Zn})$ & $(\mathrm{Pb})$ & $(\mathrm{Ni})$ & $(\mathrm{Cd})$ & $(\mathrm{Cr})$ & $\mathrm{D}$ & \\
\hline$<20(\mathrm{a})$ & $<50(\mathrm{a})$ & $<20(\mathrm{a})$ & $<20(\mathrm{a})$ & $<1(\mathrm{a})$ & $<25(\mathrm{a})$ & 0 & 0 & Não poluído \\
-30 & $-90(\mathrm{~b})$ & -30 & -30 & -2 & -50 & $0-1$ & 1 & moderado/não poluído \\
-40 & -130 & $-40(\mathrm{~b})$ & $-40(\mathrm{~b})$ & -3 & $-75(\mathrm{~b})$ & $1-2$ & 2 & Moderadamente poluído \\
$-50(\mathrm{~b})$ & -170 & $-50(\mathrm{c})$ & $-50(\mathrm{c})$ & -4 & $-100(\mathrm{c})$ & $2-3$ & 3 & forte/moderadamente poluído \\
$-60(\mathrm{c})$ & $-200(\mathrm{c})$ & -60 & -60 & -5 & -125 & $3-4$ & 4 & fortemente poluido \\
$-70(\mathrm{~d})$ & -240 & -70 & -70 & $-6(\mathrm{~b})$ & -150 & $4-5$ & 5 & extremo/fortemente poluído \\
$>70$ & $>240$ & $>70$ & $>70$ & $>6$ & $>150$ & $5-10$ & 6 & extremamente poluído \\
\hline
\end{tabular}

Cu: (a) solos não poluídos (MORSCH 1991); (b) limite moderadamente /altamente poluídos (PÁDUA 1997); (c) média de rochas sedimentares (PURVES 1975); (d) média de sedimentos argilosos (KELLER 1992). Zn: (a) limite de solos não poluídos (MORSCH 1991); (b) limite de sedimentos não poluídos (PÁDUA 1997); (c) limite de sedimentos moderado/altamente poluídos (PÁDUA 1997). Pb: (a) limite de solos não poluídos (KELLER 1992); (b) limite de sedimentos não poluídos (PÁDUA 1997); (c) limite de sedimentos moderado/altamente poluídos (PÁDUA 1997). Ni: (a) limite de sedimentos não poluídos (PÁDUA 1997); (b) limite de solos não poluídos (RUBIN 1976); (c) limite de sedimentos moderado/ altamente poluídos (PÁDUA 1997). Cd: (a) solos e sedimentos não poluídos (PÁDUA 1997); (b) limite de sedimentos moderado/altamente poluídos (PÁDUA 1997). Cr: (a) limite de sedimentos não poluídos (PÁDUA 1997); (b) limite de sedimentos moderado/altamente poluídos (PÁDUA 1997); (c) média na crosta (ALLOWAY 1990).
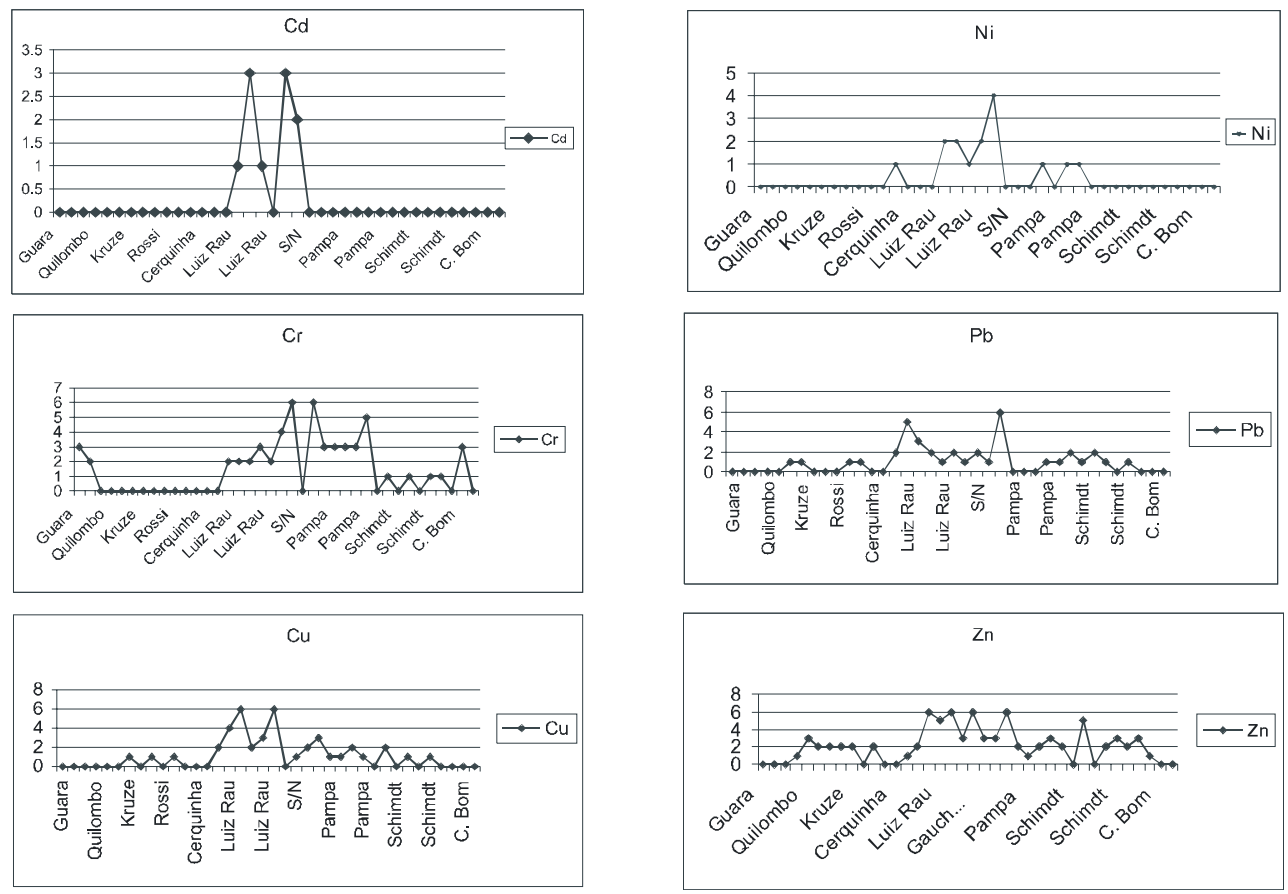

FIGURA 5 - Classes definidas na análise da Relação A (relação entre a concentração total de metais traços nos sedimentos e o nível de base estabelecido nesse estudo) para cada elemento estudado. 

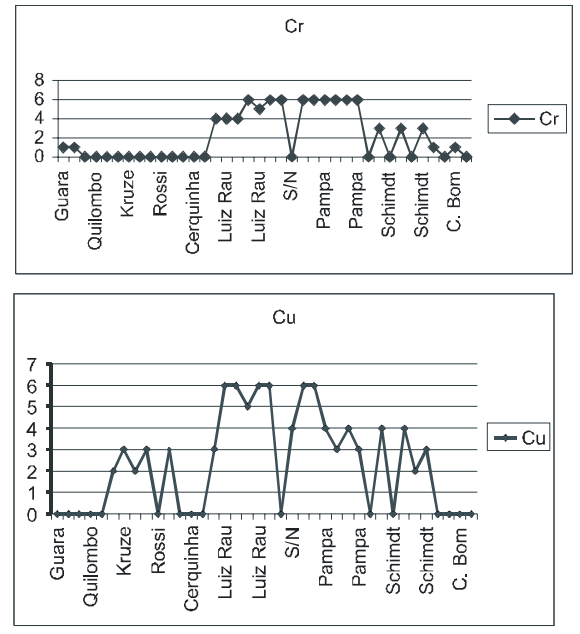
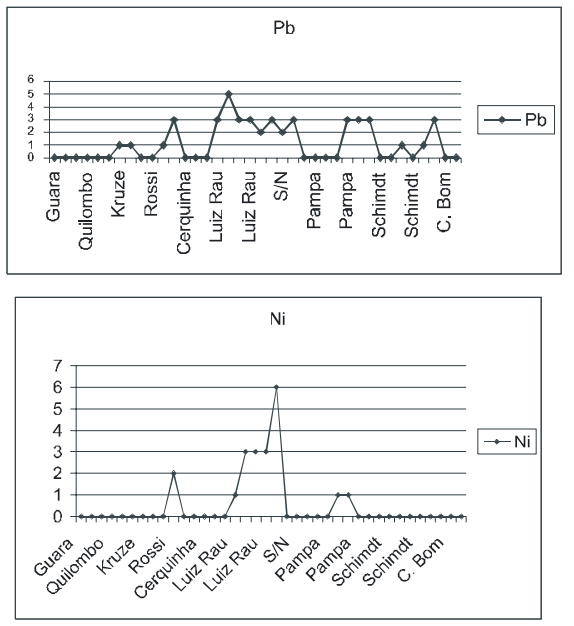

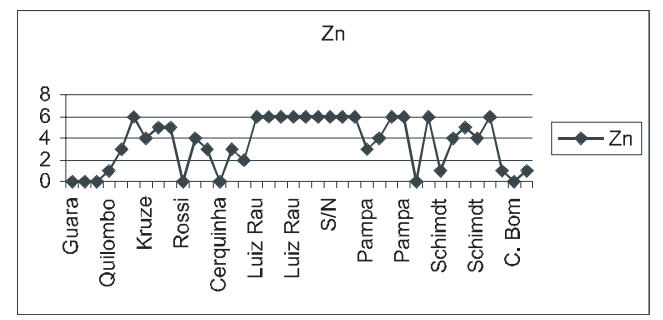

FIGURA 6 - Classes definidas na análise da Relação B (relação entre a concentração de metais traços na fração disponível dos sedimentos e o nível de base estabelecido nesse estudo) para cada elemento estudado.
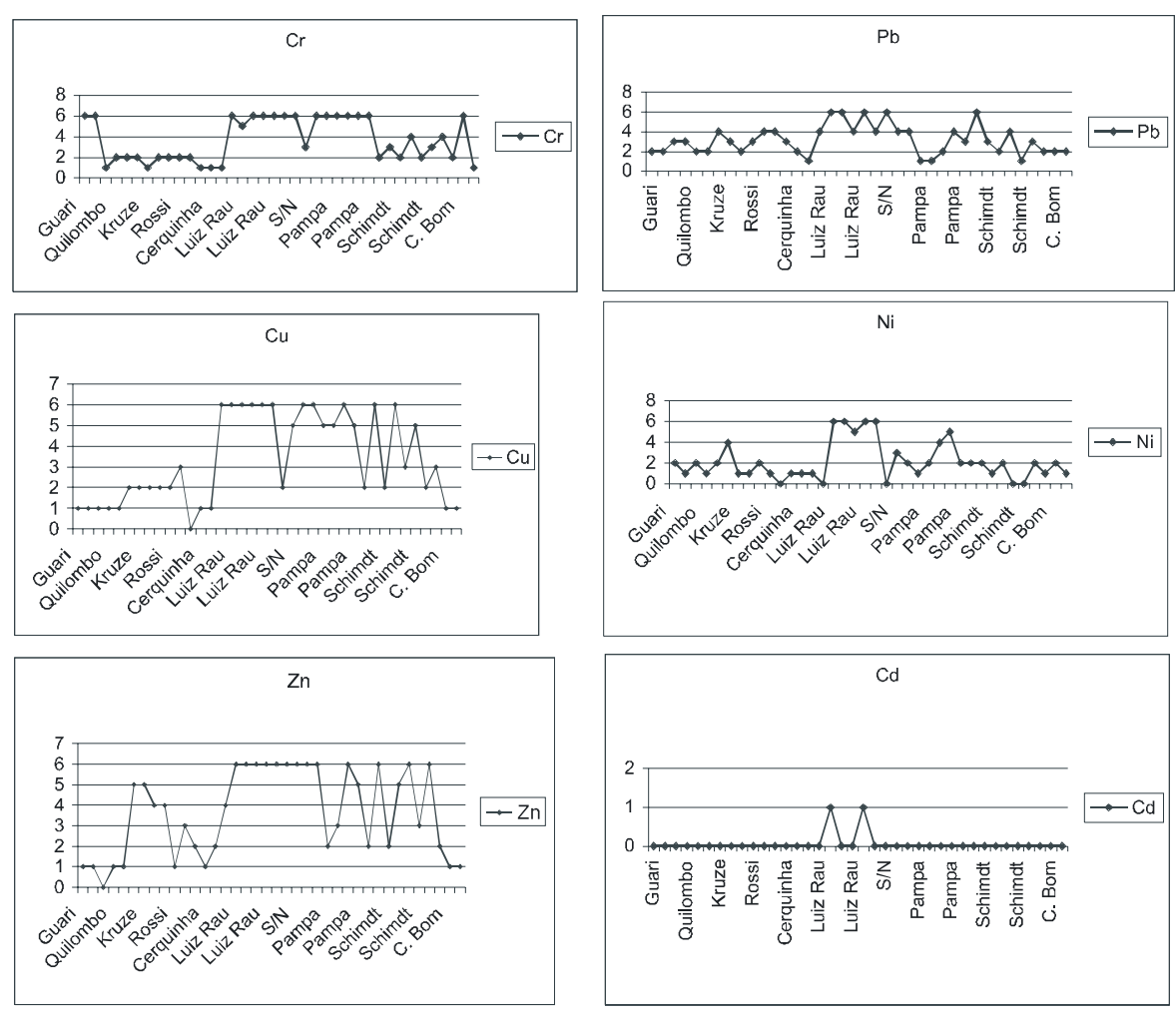

FIGURA 7 - Classes definidas na análise da Relação C (relação entre a concentração total de metais traços nos sedimentos e os teores médios para solos e sedimentos conforme dados apresentados na literatura) para cada elemento estudado. 

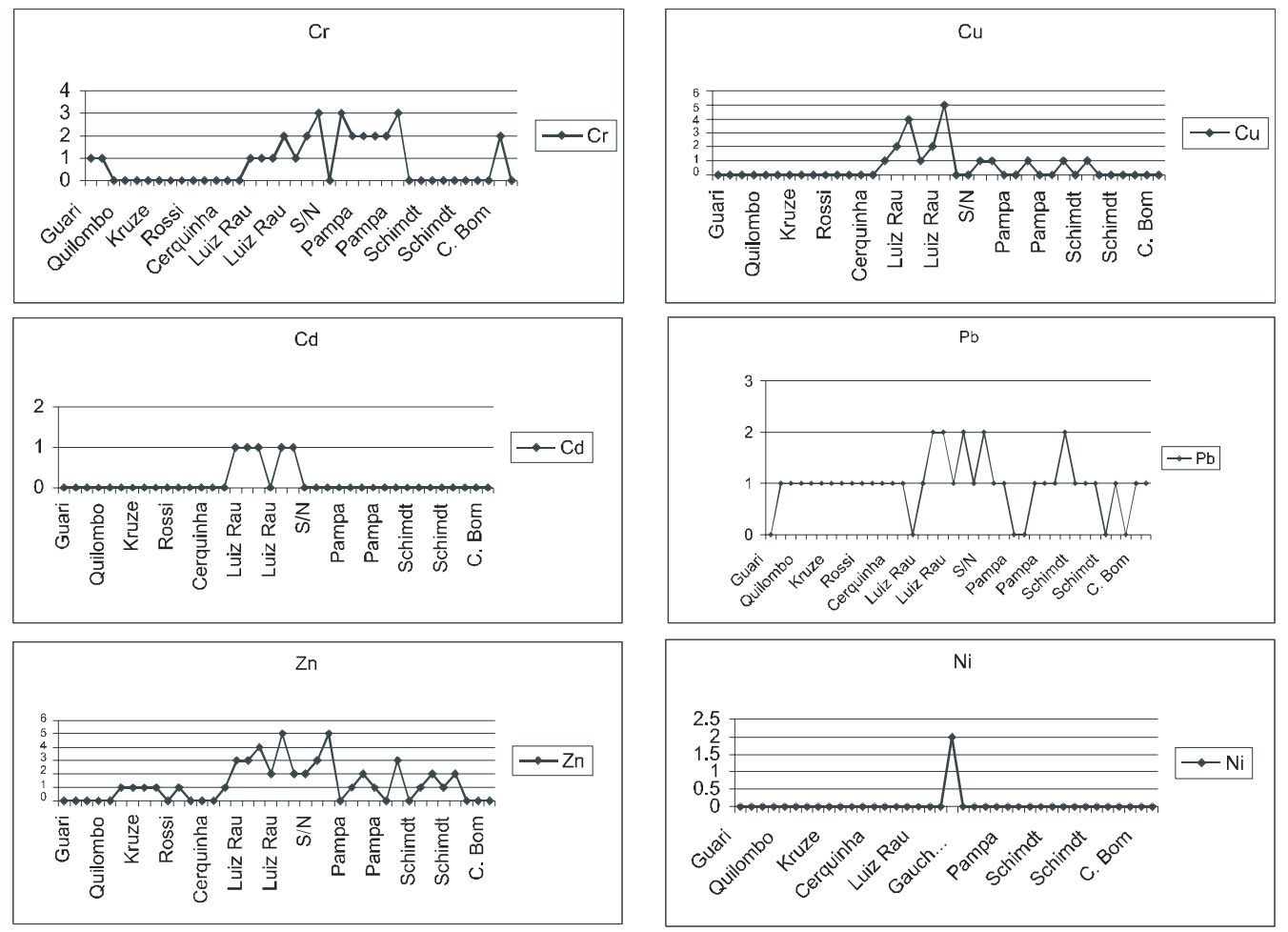

FIGURA 8 - Classes definidas na análise da Relação D (determinação do Índice de Geoacumulação - logaritmo na base 2 da concentração total de elemento, dividido pela média do valor dos folhelhos multiplicado pelo fator 1,5) para cada elemento estudado.
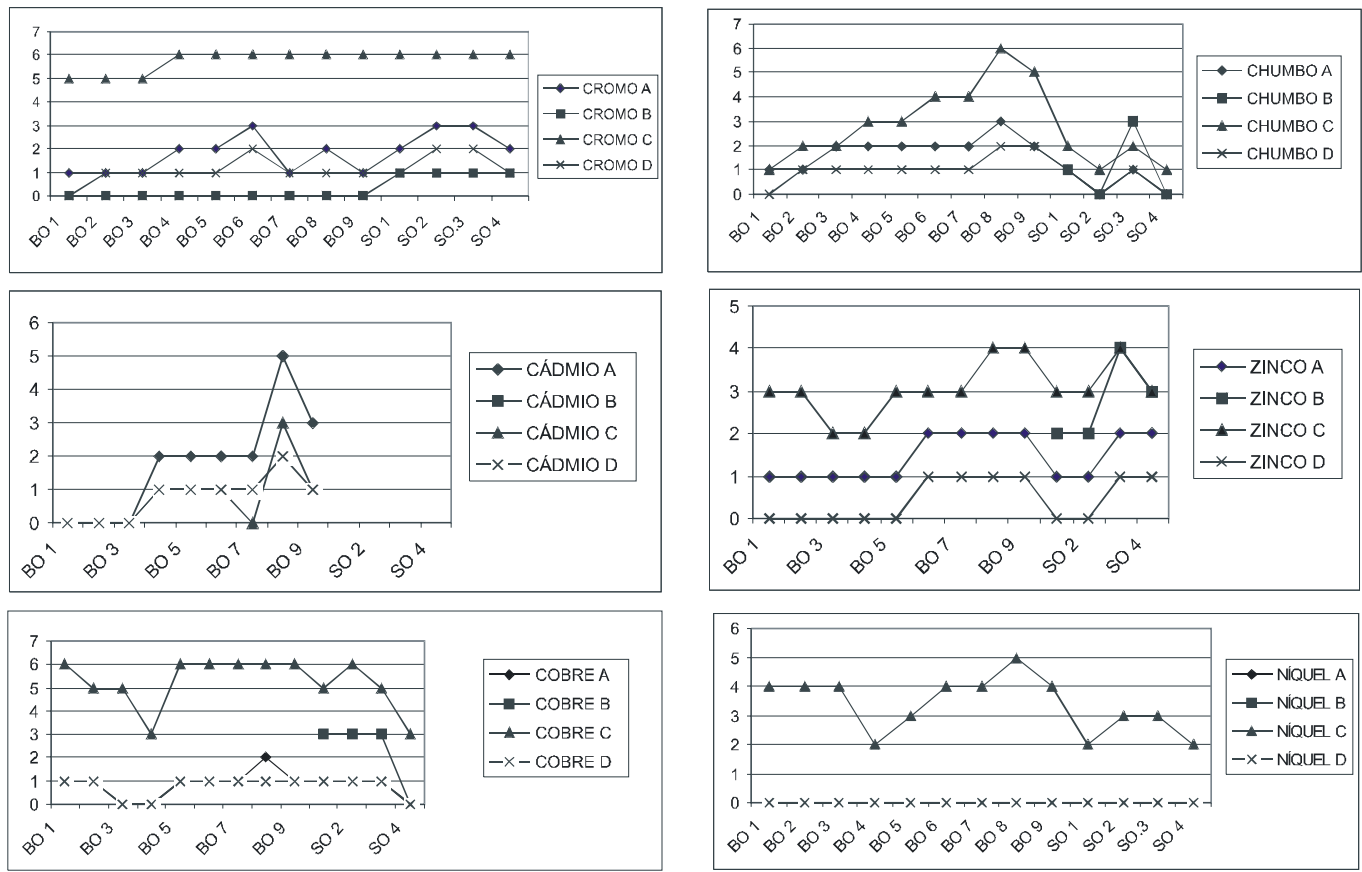

FIGURA 9 - Classes de poluição definidas pelas quatro relações para os sedimentos do Sinos. 


\subsection{Análise numérica do grau de poluição}

A análise numérica do grau de poluição é dada pelo somatório das classes obtidas em cada ponto. Nos quadros 5 e 6, temos os valores em cada ponto, para os seis elementos estudados.

O valor mais elevado para o cromo é de 21 nos arroios Pampa e Gauchinho; cobre e zinco atingem o valor máximo de 23 no Luiz Rau; chumbo atinge valor de 23 nos arroios Luiz Rau e Pampa; níquel apresenta o maior valor no Luiz Rau, de 18, e cádmio com valor máximo de 5 ocorre também no Luiz Rau.

No Rio dos Sinos, onde o valor máximo é de 18 para o grau de poluição, foram encontrados valores tão elevados como 12 para o cromo, 11 para o cobre e o zinco, 9 para o chumbo e 5 para o níquel; o cádmio, que em geral apresentou valores baixos, mostrou valor 10 em um ponto.

\section{DISCUSSÃO DO RISCO AMBIENTAL}

A partir dos valores numéricos obtidos pelo somatório das classes, temos uma análise do que denominamos risco ambiental para metais pesados, para cada elemento. A figura 3 apresenta as conclusões obtidas.

\section{Cromo}

O cromo apresenta condições de moderado risco nos pontos de coleta dos arroios Guari, Peão e no arroio Campo Bom, no ponto de coleta situado no final do curso. O arroio Luiz Rau apresenta con-

QUADRO 5 - Somatório de classes definidas nas relações de análise do grau de poluição para os arroios da área de estudo (máximo 24, 18 para o Cd, e mínimo 0)

\begin{tabular}{|c|c|c|c|c|c|c|c|c|c|c|c|c|c|c|c|}
\hline Arroios & Amostra & $\mathrm{Cr}$ & $\mathrm{Cu}$ & $\mathrm{Zn}$ & $\mathrm{Pb}$ & $\mathrm{Ni}$ & $\mathrm{Cd}$ & Arroios & Amostra & $\mathrm{Cr}$ & $\mathrm{Cu}$ & $\mathrm{Zn}$ & $\mathrm{Pb}$ & $\mathrm{Ni}$ & $\mathrm{Cd}$ \\
\hline Guari & 1 & 11 & 1 & 1 & 2 & 2 & 0 & Luiz Rau & 20 & 18 & 23 & 23 & 23 & 18 & 5 \\
\hline Peão & 2 & 10 & 1 & 1 & 1 & 1 & 0 & Gauchinho & 21 & 21 & 2 & 17 & 9 & 0 & 3 \\
\hline Quilombo & 3 & 1 & 0 & 0 & 4 & 2 & 0 & $\mathrm{~S} / \mathrm{N}$ & 22 & 3 & 10 & 17 & 17 & 3 & 0 \\
\hline Quilombo & 4 & 2 & 1 & 2 & 2 & 1 & 0 & Pampa & 23 & 21 & 15 & 21 & 9 & 2 & 0 \\
\hline Quilombo & 5 & 2 & 1 & 1 & 3 & 2 & 0 & Pampa & 24 & 17 & 15 & 23 & 23 & 1 & 0 \\
\hline Kruze & 6 & 2 & 4 & 15 & 15 & 4 & 0 & Pampa & 25 & 17 & 10 & 6 & 1 & 2 & 0 \\
\hline Kruze & 7 & 1 & 6 & 12 & 7 & 1 & 0 & Pampa & 26 & 17 & 9 & 10 & 10 & 6 & 0 \\
\hline Kruze & 8 & 2 & 4 & 12 & 12 & 1 & 0 & Pampa & 27 & 17 & 13 & 17 & 3 & 7 & 0 \\
\hline Kruze & 9 & 2 & 6 & 12 & 3 & 2 & 0 & Pampa & 28 & 20 & 9 & 14 & 9 & 2 & 0 \\
\hline Rossi & 10 & 2 & 2 & 1 & 1 & 1 & 0 & Schimdt & 29 & 2 & 2 & 6 & 4 & 2 & 0 \\
\hline J. Corrêa & 11 & 2 & 7 & 10 & 7 & 0 & 0 & Schimdt & 30 & 7 & 13 & 20 & 13 & 2 & 0 \\
\hline Cerquinha & 12 & 1 & 0 & 6 & 6 & 1 & 0 & Schimdt & 31 & 2 & 2 & 4 & 4 & 1 & 0 \\
\hline Cerquinha & 13 & 1 & 1 & 1 & 4 & 1 & 0 & Schimdt & 32 & 8 & 13 & 12 & 3 & 2 & 0 \\
\hline Manteiga & 14 & 1 & 1 & 6 & 6 & 1 & 0 & Schimdt & 33 & 2 & 5 & 16 & 7 & 0 & 0 \\
\hline Luiz Rau & 15 & 13 & 12 & 9 & 3 & 0 & 0 & Schimdt & 34 & 7 & 9 & 10 & 1 & 0 & 0 \\
\hline Luiz Rau & 16 & 12 & 18 & 21 & 21 & 9 & 2 & Schimdt & 35 & 6 & 2 & 17 & 6 & 2 & 0 \\
\hline Luiz Rau & 17 & 13 & 22 & 20 & 16 & 11 & 5 & C. Bom & 36 & 2 & 3 & 6 & 2 & 1 & 0 \\
\hline Luiz Rau & 18 & 17 & 14 & 22 & 22 & 9 & 2 & C. Bom & 37 & 12 & 1 & 1 & 3 & 2 & 0 \\
\hline Luiz Rau & 19 & 14 & 17 & 17 & 9 & 11 & 0 & B & 38 & 1 & 1 & 1 & 3 & 1 & 0 \\
\hline
\end{tabular}

QUADRO 6 - Somatório de classes definidas nas relações de análise do grau de poluição no Rio dos Sinos (máximo 18 e mínimo 0).

\begin{tabular}{|cccccccccccccc|}
\hline Ptos Sinos & $\mathrm{Cr}$ & $\mathrm{Cu}$ & $\mathrm{Zn}$ & $\mathrm{Pb}$ & $\mathrm{Ni}$ & $\mathrm{Cd}$ & Ptos Sinos & $\mathrm{Cr}$ & $\mathrm{Cu}$ & $\mathrm{Zn}$ & $\mathrm{Pb}$ & $\mathrm{Ni}$ & $\mathrm{Cd}$ \\
\hline $\mathrm{BO} 1$ & 6 & 8 & 4 & 2 & 4 & 0 & $\mathrm{BO} 8$ & 9 & 9 & 7 & 9 & 5 & 10 \\
$\mathrm{BO} 2$ & 7 & 7 & 4 & 4 & 4 & 0 & $\mathrm{BO} 9$ & 8 & 8 & 7 & 9 & 4 & 4 \\
$\mathrm{BO} 3$ & 7 & 5 & 3 & 5 & 4 & 0 & $\mathrm{SO} 1$ & 10 & 10 & 6 & 5 & 2 & \\
$\mathrm{BO} 4$ & 9 & 3 & 3 & 6 & 2 & 4 & $\mathrm{SO} 2$ & 12 & 11 & 6 & 1 & 3 & \\
BO5 & 9 & 8 & 4 & 6 & 3 & 4 & $\mathrm{SO} 3$ & 12 & 10 & 11 & 7 & 3 & \\
BO6 & 11 & 8 & 6 & 7 & 4 & 4 & $\mathrm{SO} 4$ & 10 & 3 & 9 & 1 & 2 \\
BO7 & 8 & 8 & 6 & 7 & 4 & 3 & & & & & & & \\
\hline
\end{tabular}


dições de risco moderado a alto em todos os pontos analisados.

No arroio Pampa as condições de alto risco nos trechos superiores passam a extremas no curso final. Também são extremas as condições no arroio Gauchinho, provavelmente associado ao grande número de atividades de curtume nas bacias.

Os demais arroios apresentam condições de risco que variam de baixo a muito baixo.

No Rio dos Sinos os teores de cromo nos sedimentos indicam condições de baixo risco até o trecho que recebe as águas do arroio Pampa, quando passa a moderado.

\section{Cobre}

Para o cobre, as condições de risco moderado a alto ocorrem no arroio Kruze, na margem esquerda, no arroio Schimdt, trecho médio oeste, no arroio Pampa e no arroio S/N, na margem direita. No arroio Luiz Rau as condições atingem risco extremo no ponto mais a jusante.

Nas demais drenagens da área as condições se apresentam de baixo a muito baixo risco.

No Rio dos Sinos os teores de cobre variam de baixo a moderado risco em todo trecho de estudo.

\section{Zinco}

Os teores de Zinco nos sedimentos de corrente da margem esquerda marcam condições de muito baixo risco nas drenagens que cruzam áreas não urbanas. Nas drenagens que recebem forte contribuição urbana, como os arroios Kruze e Canal João Corrêa, as condições são de risco moderado. $\mathrm{Na}$ margem direita do arroio Campo Bom há variação, de risco baixo a muito baixo. O arroio Schimdt apresenta condições de risco baixo no trecho leste, enquanto no oeste, de moderado a alto, chegando a condição extremas, na porção final. Após o encontro dos dois trechos, as concentrações passam a indicar alto risco. No arroio Pampa as condições são de risco alto a extremo em praticamente todo o curso, exceto no ponto mais a montante. Os arroios Gauchinho e $\mathrm{S} / \mathrm{N}$ apresentam condições de alto risco e o arroio Luiz Rau condições extremas a partir do trecho que recebe forte influência urbana.

Nos arroios Cerquinha e Manteiga as condições variam de muito baixo a baixo risco.

O zinco, no Rio dos Sinos, apresenta concentração nos sedimentos que indica condições de baixo risco em praticamente todo o curso, exceto na área junto ao centro da cidade de São Leopoldo, onde as concentrações indicam moderado risco.
Chumbo

No arroio Luiz Rau encontram-se condições de risco moderado a alto risco. Também no arroio Schimdt condições de risco moderado são encontradas a jusante do trecho oeste. No arroio Pampa, o trecho final apresenta condições de moderado risco.

Nos demais arroios da região as condições são de baixo a muito baixo risco.

No Rio dos Sinos, somente próximo a foz dos arroios Kruze e João Corrêa, ocorrem condições de moderado risco; no restante, as condições são de baixo a muito baixo risco.

\section{Níquel}

O risco ambiental para o niquel na área é, em geral, de baixo a muito baixo, tanto nos arroios afluentes como no Rio dos Sinos.

Somente o arroio Luiz Rau a partir do seu médio curso apresenta condições de risco moderado.

\section{Cádmio}

As concentrações de cádmio nas drenagens da área indicam condições de muito baixo risco, exceto na porção de jusante do arroio Luiz Rau, onde ocorrem condições de baixo risco.

No Rio dos Sinos as condições de muito baixo risco ocorrem até o trecho que recebe contribuição da cidade de Novo Hamburgo, quando passa para condições de baixo risco, atingindo risco moderado no ponto coletado junto à cidade de São Leopoldo.

\section{CONSIDERAÇÕES FINAIS}

Os arroios mais impactados com metais pesados são o Luiz Rau, Pampa e Gauchinho, na margem direita, e o arroio Kruze, na margem esquerda. Todos apresentam valores muito elevados de metais, em especial cromo, mas também de zinco, cobre e chumbo. Representam, portanto, as bacias de drenagem prioritárias para trabalhos de fiscalização, controle e recuperação para qualquer tentativa de minimizar a poluição na região.

\section{REFERÊNCIAS BIBLIOGRÁFICAS}

ALLOWAY, B.J. 1990. Heavy metals in soils. Blackie and Son Ltda, New York, 332p.

AXTMANN E. V. \& LUOMAS. N. 1991 Large-scale distribution of metal contamination in the finegrained sediments of the Clark Fork River. Montana. Appl. Geochem. 6 (1): 75-88. 
BAISCH, P.R.M. 1994. Les oligo-elements metalliques du systeme fluvio-lagunaire dos Patos (BRÉSIL) - Flux Devenir. Université de Bordeaux, Bordeaux, Ph.D. Thesis, 229p.

DMAE 1975. Qualidade sanitária do Rio dos Sinos. Centro de Estudos de Saneamento Básico, Relatório n.33, 77p.

FEPAM 1995. Poluição hídrica industrial: Diagnóstico de cargas poluidoras. FEPAM, Porto Alegre, 323p.

FÖRSTNER, U. \& WITTMANN, GT.W. 1983. Metal pollution in the aquatic environment. SpringerVerlag, $2^{\mathrm{a}}$ ed., $486 \mathrm{p}$.

HATJE, V.; BIDONE, E.D.; MADDOCK, J.L. 1998. Estimation of the natural and anthropogenic components of heavy metals fluxes in fresh water Sinos River, Rio Grande do Sul State, South Brazil. Enviromental Technology, 19: 483-487.

KELLER, E. 1992. Environmental geology. MacMillan Publishing Company, 6a edição, 662p.

MORSCH, V.M. 1991. Investigação do grau de contaminação dos solos e de sedimentos das regiões de Candiota e Charqueadas (RS) com elemento traço de relevância ambiental. UFSM, Santa Maria, RS, 134p.

NTEKIM, E.E.V.; EKERE, S.J; UKPONG E.E. 1993. Heavy metals distribution in sediments from Calabar River, Southeastern Nigeria. Environmental Geology, 21: 237-241.
PÁDUA, H.B. 1997. Variáveis físicas, químicas e biológicas para caracterização das águas em sistemas abertos. In: H.L. MARTOS \& N.B. MAIA (Coord.) Indicadores Ambientais, Sorocaba, SP, p.89-98.

PESTANA, M.H.D. 1989. Partição geoquímica de metais pesados em sedimentos estuarinos nas baias de Sepetiba e da Ribeira, RJ. Universidade Federal Fluminense, Instituto de Química, Niterói, Dissertação de Mestrado, 211p.

PURVES, D. 1985. Trace-elements contamination of the environment. Elsevier Science Publishers B.V., 243p.

RAMAMOORTHY, S. \& RUST, B.R. 1978. Heavy metal exchange process in sediment water systems. Environmental Geology, 2 (3):165-172.

RUBIN, A.J. 1976. Aqueous environmental chemistry of metals. Ann. Arbor Science Publishers, $2^{\mathrm{a}}$ ed., 289p.

RULE, J. 1986. Assesment of trace element geochemistry of Hampton Roads Harborand Lower Chesaspeak Bay area sediments. Environmental Geology, 8(4): 209-219.

SALOMONS, W. \& FÖRSTNER, U. 1984. Metals in the hydrocycle. Springer-Verlag, Berlin, 349p.

SINGH, M.; ANSARI, A.A.; MÜLLER, G.; SINGH, I.B. 1997. Heavy metals in freshly deposited sediments of the Gomti River (a tributary of the Ganga River): effects of human activities. Environmental Geology, 29(3/4): 246-252.

Endereço dos autores:

Luis Eduardo Robaina e Carlos Alberto da F. Pires: Universidade Federal de Santa Maria, Laboratório de Geologia Ambiental (LAGEOLAM), Departamento de Geociências, Prédio 17, Bairro Camobi, CEP $97105-$ 900, Santa Maria, RS. E-mail: lesro@hanoi.base.ufsm.br

Milton Luiz L. Formoso: Universidade Federal do Rio Grande do Sul, Instituto de Geociências, Prédio 43126, sala 208-B, CEP 91540-000, Porto Alegre.E-mail: milton.formoso@vortex.ufrgs.br 